\title{
Aplicação do Retalho Labial Superior para a Correção Cirúrgica da Hipertrofia de Pequenos Lábios
}

\author{
The Use of the Superior Labial Flap in the Surgical \\ Correction of Hypertrophy of Labia Minora
}

José Roberto Filassi, Alexandre Mendonça Munhoz, Marcos Desidério Ricci, Nilson Roberto de Melo

\section{RESUMO}

\begin{abstract}
Objetivos: descrever técnica cirúrgica para tratamento da hipertrofia de pequenos lábios com a utilização do retalho labial superior.

Métodos: no periodo de maio de 1998 a julho de 2002, 10 pacientes com hipertrofia de pequenos lábios foram submetidas à redução do excesso pela ressecção do segmento inferior com incisão em " $L$ ". A incisão transversal vai da borda externa do lábio em direção à carúncula himenal e a incisão longitudinal parte dali até próximo à fúrcula. A borda do retalho superior é rebaixada para se fechar o defeito causado pela ressecção inferior.

Resultados: com periodo médio de 45 dias após a cirurgia as pacientes estavam satisfeitas com o aspecto estético e funcional. Não houve mudança na sensibilidade local. Apenas 2 casos apresentaram complicações: um caso de equimose perineal e outro com deiscência unilateral parcial precoce do retalho. Não foram observados casos de infecção, necrose e deiscência tardia.

Conclusão: a utilização do retalho superior para correção de hipertrofia de pequenos lábios produz bom resultado estético e funcional, com poucas complicações e de fácil resolução.
\end{abstract}

PALAVRAS-CHAVE: Pequenos lábios. Hipertrofia. Cirurgia: complicações.

\section{Introdução}

A hipertrofia dos pequenos lábios é causa comum de desconforto funcional, podendo limitar a atividade sexual e fisica, além de motivar queixa estética. Na maioria dos casos, a hipertrofia não apresenta etiologia definida, sendo considerada constitucional. Em raros casos, a hipertrofia pode ser secundária a insuficiência de drenagem linfática, alterações hormonais e traumas externos na região vulvar ${ }^{1,2}$.

Hospital Sírio Libanês - São Paulo-SP

Correspondência :

José Roberto Filassi

Rua Adma Jafet, $n^{\circ} 50-14^{\circ}$ andar.

01308-050 - São Paulo - SP

Telefone/FAX: (011) 3231-0108

email: desiderion@uol.com.br
Na literatura, o relato de séries clínicas avaliando o diagnóstico e forma de tratamento é escasso, e quase sempre o manejo é cirúrgico.

Nos últimos anos, e principalmente a partir da década de 90 , a cirurgia estética da região vulvar apresentou grande evolução. As técnicas anteriormente descritas, com resultados estéticos desanimadores, e baseadas no conceito da amputação completa dos pequenos lábios, evoluíram para procedimentos mais conservadores. Estas técnicas deixam a cicatriz em tal posição, que terminam por preservar a unidade anatômica da vulva, propiciando resultados estéticos e funcionais mais satisfatórios ${ }^{3-6}$.

Os autores, por meio do planejamento préoperatório individualizado e sem a utilização de demarcações rígidas nas ressecções, descrevem o tratamento cirúrgico das hipertrofias de pequenos lábios pela reconstrução com o retalho cutâneo de pedículo superior. 


\section{Métodos}

No período de maio de 1998 a julho de 2002, dez pacientes portadoras de hipertrofia de pequenos lábios foram submetidas à cirurgia de ressecção do excesso de tecido com reconstrução imediata, utilizando o retalho remanescente superior, no Hospital Sírio Libanês de São Paulo. Todas as pacientes apresentaram a queixa estética, com maior ou menor grau de importância, como o motivo principal que levou à procura por tratamento. Esta queixa era associada a limitação para atividade sexual em seis pacientes e restrição em determinados exercícios físicos em quatro.

Em algumas situações foram realizadas cirurgias associadas, sendo três casos de perineoplastia e dois de histerectomia abdominal.

Todas as pacientes foram submetidas ao exame clínico mamário e ginecológico, Papanicolaou da ecto e endocérvice, vulvoscopia, colposcopia e ultra-sonografia endovaginal. Com base nos dados de história clínica, em todos os casos foram afastadas etiologias infecciosas e hormonais, sendo a casuística representada por hipertrofias de natureza idiopática constitucional. O trabalho foi aprovado pela Comissão de Ética e Pesquisa Médica do Hospital Sírio Libanês, de São Paulo.

Todas as pacientes foram submetidas ao tratamento cirúrgico sob anestesia local associada à sedação. Nas pacientes com cirurgias pélvicas associadas foram empregados o bloqueio anestésico peridural e sedação.

A técnica cirúrgica tem início com a paciente em posição ginecológica, quando se realizam o reconhecimento e demarcação dos parâmetros anatômicos principais na face interna e externa dos pequenos lábios.

A primeira marcação na região vulvar, denominada ponto "A", representa o limite inferior do vetor vertical de ressecção (Figura 1). Em condições ideais, este ponto deve situar-se junto à transição com a mucosa vaginal e, diferentemente dos pontos seguintes, se apresenta como parâmetro fixo.

Com o emprego da pinça cirúrgica, realizase a tração para baixo dos pequenos lábios a partir da sua porção média. Este passo é fundamental e por meio da união deste local com o ponto "A", previamente demarcado, avalia-se o grau de flacidez cutânea, o excesso de pele, tecido subcutâneo, mucosa e principalmente o aspecto estético desejado. Na ausência de tensão, após a união destes pontos e a correção satisfatória do excesso de tecido, denomina-se o ponto de tração realizado pela pinça como ponto "B".

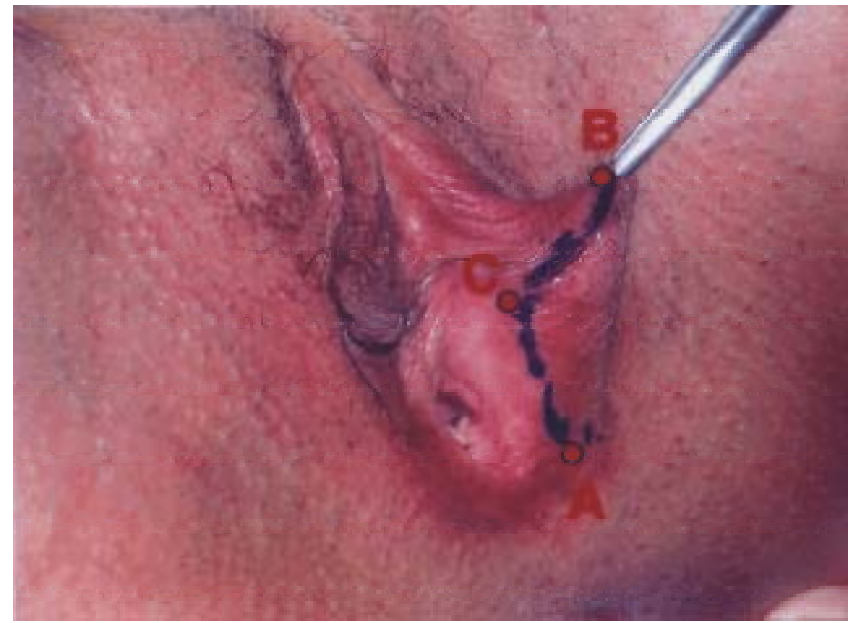

Figura 1 - Esquema demonstrando os parâmetros anatômicos principais na demarcação da área de ressecção. Notam-se os pontos "A", "B" e "C" e os vetores vertical ("A" + "C") e horizontal ("B" + "C").

Na região próxima à transição com a mucosa vaginal, alinhando com o ponto "A", se realiza a demarcação do ponto "C". O posicionamento deste ponto, quanto à distância até o ponto "A", é avaliada de acordo com o excesso de pele e mucosa após a tração inferior da pinça.

Nas hipertrofias acentuadas, há a necessidade de ressecções maiores e, portanto, o posicionamento do ponto " $\mathrm{C}$ " termina por se localizar em região superior ao ponto "B". Nas hipertrofias de menor volume, o ponto "C" pode se localizar em posição inferior ao ponto "B", tendo assim margem de ressecção menor.

Denomina-se vetor vertical a união do ponto " $\mathrm{A}$ " com o ponto "C", e vetor horizontal, a união do ponto "B" com o ponto "C".

Estes vetores devem ser demarcados na face externa (lateral) e na face interna (medial) do pequeno lábio. A área de ressecção é definida neste momento pela região demarcada pelos vetores horizontais e verticais descritos acima. Após a marcação dos parâmetros principais, e a delimitação da área de ressecção, se realiza a infiltração dos pequenos lábios, principalmente no local das incisões, com solução de cloridrato de lidocaína $2 \%(20 \mathrm{~mL})$, marcaina $1 \%(20 \mathrm{~mL})$, adrenalina 1:1000 (1 ampola) e soro fisiológico $(60 \mathrm{~mL})$. Com esta solução, além da analgesia pós-operatória prolongada, se conseguem menor sangramento e edema pelos efeitos de vasoconstricção promovidos pela adrenalina.

Realiza-se a incisão das linhas demarcadas com bisturi lâmina 15, e em seguida a ressecção do excedente de pele e mucosa.

Após a completa ressecção, realiza-se a hemostasia local e em seguida a sutura do ponto "B" com o ponto "A". Neste sentido, promove-se a 
tração inferior do retalho cutâneo-mucoso remanescente (Figuras 2 e 3 ).

Com objetivo de reforçar a sutura, esta deve ser feita em dois ou mais pontos com fios absorviveis entre o tecido subcutâneo do retalho e a área cruenta do vetor vertical. Em seguida, utilizando também fios absorviveis, realiza-se a sutura dos planos cutâneos e mucosos com pontos separados.

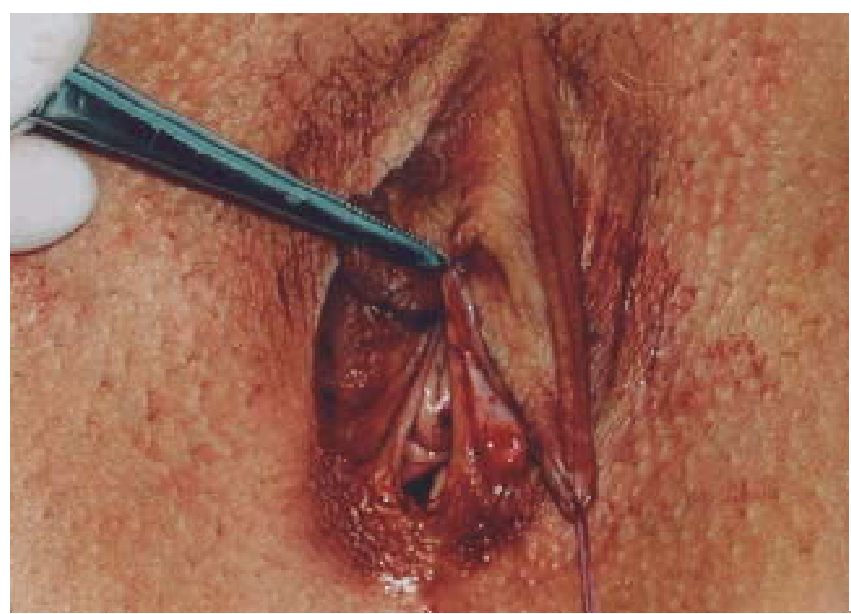

Figura 2 - Intra-operatório demonstrando o retalho remanescente superior e o sentido de tração em direção inferior, com objetivo de união dos pontos "A" e "B".

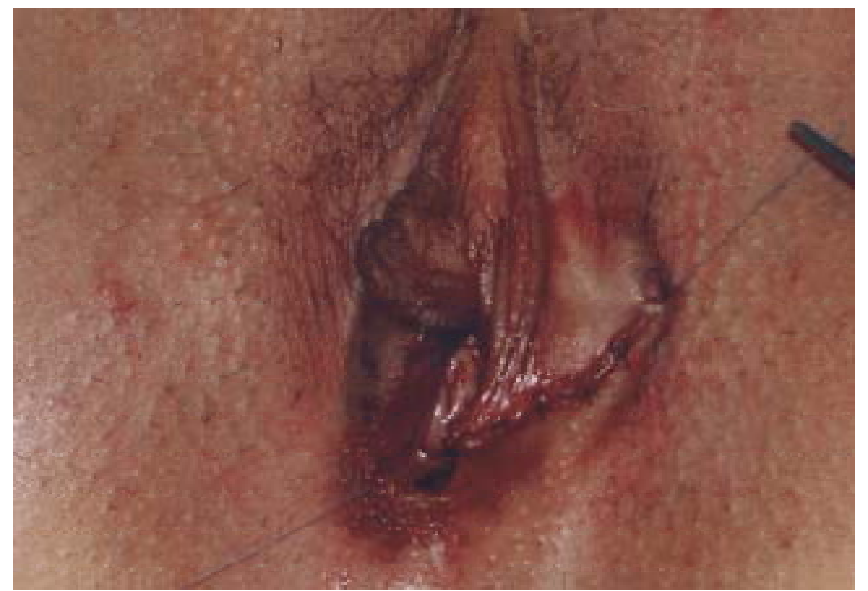

Figura 3 - Intra-operatório demonstrando o aspecto da sutura com pontos absorviveis separados.

\section{Resultados}

O tempo médio de cirurgia foi de 40 minutos, e as pacientes receberam alta hospitalar com um dia de internação, exceto aquelas submetidas a histerectomia, para as quais a permanência hospitalar foi de dois dias. A antibioticoterapia profilática foi realizada com cefalosporina de segunda geração, instituída imediatamente antes da cirurgia e mantida até 36 horas no pós-operatório.
Como complicações houve um caso de equimose acentuada da região perineal, na qual a conduta foi expectante, e um caso de deiscência precoce unilateral. Neste caso houve a necessidade de reintervenção cirúrgica unilateral em período posterior. Não foram observados casos de infecção, necrose do retalho ou deiscências tardias.

Em todos os casos observou-se edema pósoperatório da região vulvar em maior ou menor intensidade.

Após período médio de 45 dias, todas as pacientes foram interrogadas sobre a aparência da genitália externa e a resolução dos problemas originais, sendo que todas se apresentaram satisfeitas do ponto de vista estético e funcional em relação às queixas pré-operatórias. Nenhuma paciente apresentou desconforto na relação sexual, sendo esta prática autorizada após 4 semanas da cirurgia. Não foram relatadas alterações de sensibilidade na região operada.

Ao exame físico, realizado nesta época, as cicatrizes apresentavam-se bem posicionadas e quase imperceptiveis à inspeção clínica.

\section{Discussão}

A hipertrofia dos pequenos lábios é condição clínica pouco freqüente, provavelmente por ser queixa não valorizada pelos ginecologistas. Sua etiologia, na maioria dos casos, é constitucional, sem causa definida. Em raras situações pode se correlacionar com o bloqueio da drenagem linfática da vulva, como ocorre na infecção por Filaria sanguinis hominis, agente etiológico da filariose. Outras causas, igualmente incomuns, relatadas na literatura, decorrem da estimulação hormonal por administração de androgênios durante a infância, traumas repetidos na região vulvar e tração progressiva dos pequenos lábios, característica de algumas culturas indigenas ${ }^{1,2}$.

O excesso de projeção dos pequenos lábios, sobre os grandes lábios, pode em algumas situações causar desconforto físico e psicológico. A dor ou incômodo na relação sexual, irritação local, dificuldade para prática de alguns esportes e motivos estéticos são as queixas principais observadas em séries clínicas ${ }^{2,3,7}$.

A definição de hipertrofia de pequenos lábios não é bem clara na literatura e são escassos os relatos que definem parâmetros clínicos de diagnóstico e conduta cirúrgica.

Alguns autores definem hipertrofia quando a distância entre a projeção máxima e a base do lábio menor excede quatro centímetros de com- 
primento. Na maioria dos casos se manifesta de maneira assimétrica, podendo ocorrer também a hipertrofia bilateral e unilateral ${ }^{3,8}$.

Friedrich $^{9}$, em 1983, utiliza os mesmos parâmetros de mensuração, porém cita o limite de até cinco centímetros de extensão como critério para diagnóstico de hipertrofia. Segundo o autor, esta mensuração deve ser realizada por meio da visualização lateral, mantendo o minimo de tração durante a leitura da medida.

Não há consenso em relação ao diagnóstico objetivo e à mensuração da intensidade da hipertrofia, fatos estes considerados importantes no estabelecimento de protocolos de tratamento e eventual indicação da cirurgia reparadora.

A aplicação de medidas ou conceitos habitualmente utilizados em indicações cirúrgicas não tem aplicação prática no tratamento da hipertrofia de pequenos lábios. Nesta situação, a ausência de parâmetros claros e reprodutíveis torna fundamental o bom senso do profissional na indicação e contra-indicação do procedimento, uma vez que, tratando-se de queixas subjetivas e de natureza estética, a indicação da cirurgia se baseia na vontade da paciente.

A avaliação do excesso de pele e flacidez, a dificuldade para relação sexual e a limitação funcional à deambulação e prática de esportes são fatores importantes na indicação da redução de pequenos lábios.

O tratamento cirúrgico é descrito desde a antiguidade, e relatado em sociedades islâmicas como conceito de purificação. Nestas culturas a amputação dos pequenos lábios é descrita como circuncisão feminina e apresenta motivos puramente religiosos. Aventa-se que, nestas populações, dois terços das mulheres são circuncisadas, sendo a maioria dos procedimentos representados por amputações completas dos pequenos lábios, realizadas pela própria população leiga ${ }^{4,10}$.

Até recentemente, a grande maioria das técnicas cirúrgicas estavam relacionadas à ressecção elíptica de toda a extensão do pequeno lábio que se apresentasse em excesso ${ }^{3,7}$. Estas técnicas determinavam maior trauma cirúrgico e resultados estéticos pouco satisfatórios, como a colocação da cicatriz final sobre a margem remanescente dos pequenos lábios. A presença da linha irregular de sutura e a perda do contorno natural são as principais desvantagens estéticas destas técnicas ${ }^{5}$. Além disto, do ponto de vista funcional, como a incisão cirúrgica fica adjacente ao clitóris, há risco de interferir na sensibilidade local. Esta técnica é uma das mais realizadas e difundidas pelos ginecologistas. Talvez, pelos resultados pouco satisfatórios, a queixa de hipertrofia de pequenos lábios é quase sempre minimizada ou esquecida durante a anamnese.

Assim, alguns autores, a partir do final da década de 90, sugerem novas propostas de tratamento, modificando as incisões cirúrgicas previamente descritas ${ }^{3-6}$.

Alter ${ }^{5}$, em 1998, propõe a ressecção central dos pequenos lábios e aproximação primária dos retalhos superiores e inferiores remanescentes. Pela experiência clínica inicial de quatro casos, o autor enfatiza a preservação da margem do pequeno lábio como fator importante na obtenção do resultado estético mais natural.

Maas e Hage ${ }^{6}$, em 1999, enfatizam a importância de evitar incisões lineares sobre a margem dos pequenos lábios, uma vez que a retração cicatricial resultaria em redução do intróito vaginal. Assim, os autores propõem, com resultados favoráveis em 13 pacientes, a incisão em forma de "linha quebrada" à semelhança da "plástica em W", habitualmente realizada para tratamento de bridas cutâneas.

Em 2000, Choi e $\mathrm{Kim}^{4}$, seguindo a mesma tendência dos autores precedentes, descrevem a correção da hipertrofia de pequenos lábios sem a utilização de incisões externas. Pela ressecção de pele da região central, na face interna dos pequenos lábios, seguida pela aproximação primária das margens, os autores enfatizam a preservação da anatomia externa dos pequenos lábios e a manutenção da coloração e contorno da região.

Rouzier et al. ${ }^{3}$, em 2000, descrevem a ressecção do excesso de pequenos lábios em forma de "V" na região do terço inferior, e a manutenção do retalho cutâneo-mucoso remanescente através do pedículo vascular superior. Com casuística de 163 casos, e por meio de questionários enviados às pacientes, os autores descrevem $89 \%$ de melhora estética e 93\% de melhora funcional após a cirurgia. Como complicações, em $7 \%$ dos casos houve a necessidade de reintervenção cirúrgica secundária à deiscência da sutura.

A individualização do tratamento é fundamental, uma vez que a apresentação clínica e sobretudo a extensão de tecido em excesso são extremamente variadas. Na mesma paciente, dificilmente encontramos simetria dos pequenos lábios; além disto, cada caso é completamente distinto, o que impossibilita o uso de técnicas rígidas pré-estabelecidas. Neste sentido, a programação pré-operatória e marcação dos pontos "A", "B" e "C", lateral e medial bilateral, sem demarcação rígida, permite melhor adequação da extensão de ressecção à quantidade de tecido excedente.

A flacidez cutânea, a quantidade de tecido subcutâneo, o excesso de pele e mucosa e o compri- 
mento da margem vulvar constituem fatores importantes neste planejamento. A possibilidade de programação da incisão, com base na ponderação destes fatores, permite maior beneficio estético e funcional para as pacientes, além de reduzir acentuadamente as complicações a curto e longo prazo.

\section{ABSTRACT}

Purpose: to describe the use of a superior labial flap for the treatment of labia minora hypertrophy.

Methods: from May, 1998 to July, 200210 patients with labia minora hypertrophy were submitted to reduction of the labial excess through the resection of the inferior segment by an L-shaped incision. The transversal incision was done starting on the labial external border towards the hymenal caruncles, and the longitudinal incision, from that point until near the furcula. The border of the superior flap was then lowered to eliminate the defect caused by the inferior resection.

Results: within an average period of 45 days after surgery, the patients were satisfied with its esthetical and functional aspects. The local sensibility did not change. Only two cases presented complications: one case with perineal ecchymosis and the other with partial, early unilateral dehiscense. Infection, necrosis and late dehiscense were not observed. Conclusion: the utilization of the superior flap in the correction of labia minora hypertrophy produces a satisfactory esthetical and functional result with few complications and easy resolution.

KEYWORDS: Labia minora. Hypertrophy. Surgery: complications.

\section{Referências}

1. Radman HM. Hypertrophy of the labia minora. Obstet Gynecol 1976; 48 Suppl:78S-79S.

2. Chavis WM, LaFerla JJ, Niccolini R. Plastic repair of elongated, hypertrophic labia minora. J Reprod Med 1989; 34:374-5.

3. Rouzier R, Louis-Sylvestre C, Paniel B, Haddad B. Hypertrophy of labia minora: experience with 163 reductions. Am J Obstet Gynecol 2000; 182:35-40.

4. Choi HY, Kim KT. A new method for aesthetic reduction of labia minora (The deepithelialized reduction labioplasty). Plast Reconstr Surg 2000; 105:419-22.

5. Alter GJ. A new technique for aesthetic labia minora reduction. Ann Plast Surg 1998; 40:287-90.

6. Maas SM, Hage JJ. Functional and aesthetic labia minora reduction. Plast Reconstr Surg 1999; 105:1453-6.

7. Hodgkinson DJ, Hait G. Aesthetic vaginal labioplasty. Plast Reconstr Surg 1983; 74:414-6.

8. Jeffcoate N. Hypertrophy of the labia minora: spaniel ear nymphae. In: Tindall VR, editor. Principles of Gynecology. $4^{\text {th }}$ ed. London: Butterworths; 1975. p. 151.

9. Friedrich EG. Vulvar disease. $2^{\text {nd }}$ ed. Philadelphia: WB Saunders; 1983. p. 86.

10.Assaad MB. Female circumcision in Egypt: social implications, current research, and prospects for change. Stud Fam Plann 1980; 11:3-16.

Recebido em: $23 / 7 / 03$ Aceito com modificações em: 19/10/04 